\title{
Plasma distribution of non-cholesterol sterols precursors of cholesterol synthesis and phytosterols depend on HDL concentration
}

Valeria Sutti Nunes ( $\nabla$ valeriasutti@gmail.com )

Hospital das Clinicas HCFMUSP, Faculdade de Medicina, Universidade de Sao Paulo https://orcid.org/0000-0002-6769-5412

Eliton Juniro Silva

Universidade de Sao Paulo

Guilherme Silva Ferreira

Universidade de Sao Paulo

Sayonara Ivana Santos Assis

Universidade de Sao Paulo

Vanessa Helena de Souza Zago

Universidade Estadual de Campinas

Patrícia Miralda Cazita

Universidade de Sao Paulo

Edna Regina Nakandakare

Universidade de Sao Paulo

Eliana Cota Faria

Universidade Estadual de Campinas

Eder Carlos Rocha Quintão

Universidade de Sao Paulo

Research

Keywords: 24-hydroxycholesterol, 27-hydroxycholesterol, desmosterol, lathosterol, campesterol, sistosterol, very-low-density lipoproteins, low-density lipoproteins, high-density lipoproteins

Posted Date: April 23rd, 2020

DOI: https://doi.org/10.21203/rs.3.rs-22905/v1

License: (c) (i) This work is licensed under a Creative Commons Attribution 4.0 International License.

Read Full License 


\section{Abstract \\ Background}

Because several sterols are carried by serum lipoproteins that are pivotal in cholesterol metabolism and atherosclerosis this study aimed at investigating their lipoprotein distribution in individuals differing according to HDL-C plasma levels.

\section{Methods}

Healthy participants were selected with plasma HDL-C concentrations $<40 \mathrm{mg} / \mathrm{dL}$ (Low HDL, $\mathrm{n}=12$ ), above $40 \mathrm{mg} / \mathrm{dL}$ and below $60 \mathrm{mg} / \mathrm{dL}$ (Controls, $\mathrm{n}=10$ ), or above $60 \mathrm{mg} / \mathrm{dL}$ (High HDL, $\mathrm{n}=15$ ). Plasma lipoproteins were separated by sequential ultracentrifugation. [27-OHC]), noncholesterol sterols precursors of cholesterol synthesis (NCSPCS: desmosterol and lathosterol) and phytosterols (campesterol and sitosterol) were measured by GC-MS analysis. Diferences between groups were compared by Kruskall-walys and Dunn's post-test with correction by Bonferroni.

\section{Results}

The groups were similar in about BMI, age, gender distribution and blood glucose, total cholesterol and LDL-C. The High HDL group had lower concentrations of triglycerides and VLDL-C compared to others. Percentage distribution of plasma $24-\mathrm{OHC}$ and $27-\mathrm{OHC}$ in lipoproteins did not differ among investigated groups, but plasma concentration of 24-OHC was lower in High HDL than in Low HDL (27- OHC significance difference was borderline). NCSPCS and phytosterols predominate in LDL, which carries approximately $50 \%$ of these molecules. Approximately $30 \%$ of desmosterol and lathosterol are present in $\mathrm{HDL}$, the High HDL group carrying higher percent of both sterols than the Low HDL and Control groups. Less than $20 \%$ of NCSPCS are transported in VLDL. Concentration of plasma campesterol and sitosterol did not differ among groups. However, LDL sitosterol percent distribution is lower in High HDL than in Low HDL and Controls. Campesterol and sitosterol percent distributions are higher in High HDL than in the Low HDL group.

\section{Conclusions}

Elevated NCSPCS in the High HDL group suggests HDL facilitates the export of these sterols from cells but not the export of the cholesterol metabolite 24-OHC which is lower in the High HDL than in the Low HDL group. Percent campesterol and sitosterol elevated in HDL suggests phytosterols absorbed in the enterocytes are incorporated mostly into the nascent HDL.

\section{Introduction}


Noncholesterol sterols precursors of cholesterol synthesis (NCSPCS: desmosterol and lathosterol, and squalene) are carried by serum lipoproteins and reflect cholesterol synthesis rates, whereas plant sterols, campesterol and sitosterol, and cholestanol, a metabolite of cholesterol, reflect the efficiency of cholesterol absorption in normal and hyperlipidemic populations [1-3]. Furthermore, Nunes and collaborators demonstrated that individuals with high HDL-C plasma levels have greater plasma concentrations of cholesterol absorption markers (campesterol and $\beta$-sitosterol) and lower plasma concentration of lathosterol which is a marker of body cholesterol synthesis [4].

Oxysterols, in addition to contributing directly to hepatic cholesterol excretion through bile acid formation, also have anti-atherosclerotic effects contributing to eliminate the excess of celular cholesterol via ABCA1 or passive diffusion. 24-hydroxycholesterol (24-OHC) and 27-hydroxycholesterol (27-OHC) are transported mainly in LDL and HDL. Babiker et al. investigated the distribution of 24-OHC, 27-OHC and $3 \beta$-hydroxy-5-cholestenoic acid in lipoprotein fractions and lipoprotein-free plasma of seven healthy nonsmokers volunteers showing that $24-\mathrm{OHC}$ and $27-\mathrm{OHC}$ have similar pattern of distribution in lipoproteins (40\% being present in LDL and $40-50 \%$ in $\mathrm{HDL}$ ). The $27-\mathrm{OHC} /$ cholesterol and $24-\mathrm{OHC} /$ cholesterol ratios were higher in the HDL fraction, indicating that HDL may be important for the transport of these oxysterols [5]. In this regard, increased plasma $27-\mathrm{OHC} /$ cholesterol ratio of individuals with low HDL-C when compared to those with high HDL-C, suggests cellular cholesterol excretion higher via this pathway, thus protecting cells from the cholesterol accumulation [6].

Due to the importance of HDL in the reverse cholesterol transport pathway the present study aimed at investigating the lipoprotein distribution of oxysterols, noncholesterol sterol precursors of cholesterol synthesis (NCSPCS) and phytostrols in individuals that differed according to HDL-C plasma levels.

\section{Materials And Methods}

\section{Subjects}

Volunteers of both genders were recruited from primary health care centers in Campinas (SP-Brazil) and Ambulatório de Dislipidemia do Serviço de Endocrinologia e Metabologia do Hospital das Clínicas da Faculdade de Medicina da Universidade de São Paulo. The study was approved by the Research Ethics Committee of UNICAMP School of Medicine under n 120/2007 and Hospital das Clínicas da Faculdade de Medicina da Universidade de São Paulo under nº 149/7. All participants were informed of the objectives of the protocol and signed an informed written consent according to research protocols approved by the Ethics Committee of HCFMUSP and UNICAMP.

The study included nonsmokers, asymptomatic individuals with body mass index (BMI) lower than $30 \mathrm{~kg} / \mathrm{m} 2$, without regular use of any medications that interfere with lipid metabolism and daily intake of alcohol lower than $14 \mathrm{~g}$, as previously described [7].

Subjects were selected with plasma HDL-C concentrations below $40 \mathrm{mg} / \mathrm{dL}$ (Low HDL, 7 males and 5 females), above $40 \mathrm{mg} / \mathrm{dL}$ and below $60 \mathrm{mg} / \mathrm{dL}$ (Control, 4 males and 6 females), or above $60 \mathrm{mg} / \mathrm{dL}$ 
(High HDL, 7males and 8 females) corresponding to values below the 10 th percentile and above the 90th percentile of the Brazilian population. The subjects' ages were between 20-74 years and body mass index (BMI) below $30 \mathrm{~kg} / \mathrm{m}^{2}$. The exclusion criteria were obesity, diabetes mellitus, metabolic syndrome, thyroid function disorders, liver and kidney failures, smoking, alcohol abuse and use of medications that might interfere with the metabolism of cholesterol.

\section{Study Procedures}

Blood was drawn after $12 \mathrm{~h}$ fasting period into tubes containing ethylenediamine tetraacetic acid (EDTA) (10\%). Plasma was immediately separated and added benzamidine $2 \mathrm{mM}(5 \mu \mathrm{L} / \mathrm{mL})$, gentamycin + cloranphenicol $15 \mathrm{mM}(20 \mu \mathrm{L} / \mathrm{mL})$, phenyllmethyl sulfonil fluoride $0,5 \mathrm{mM}(0,5 \mu \mathrm{L} / \mathrm{mL})$ and aprotinin $10 \mathrm{mg} / \mathrm{mL}(5 \mu \mathrm{L} / \mathrm{mL})$ and butylated hydroxytoluene (BHT). Plasma lipoproteins very-low-density lipoproteins (VLDL), low-density lipoproteins (LDL), high-density lipoproteins (HDL) and lipid free fraction (LFF) were separated by sequential ultracentrifugation using a Beckman Model L-8 ultracentrifuge $50 \mathrm{Ti}$ rotor (Beckman Instruments, Palo Alto, CA, USA) [8]. Plasma and lipoprotein NCSPCS (desmosterol, lathosterol) and phytosterols (campesterol and sitosterol) were measured in samples $(100 \mu \mathrm{L})$ added $5 \mathrm{a}-$ cholestane $(1 \mu \mathrm{g})$ as the internal standard, hydrolyzed with $\mathrm{KOH}$ in ethanol $(1 \mathrm{~mol} / \mathrm{l}, 1 \mathrm{ml})$ at $60{ }^{\circ} \mathrm{C}(1 \mathrm{~h})$ and extracted with hexane. Sterols were derivatized with a sylilating solution (pyridine and BSTFA (N,Obis (trimethylsilyl) trifluoroacetamide) + 1\% TMCS (trimethylchlorosilane) (1:1, v/v) (Supelco 33155-U) for $1 \mathrm{~h}$ at $60^{\circ} \mathrm{C}[4]$

Plasma and lipoproteins oxysterols (24-OHC and 27-OHC) were measured according S. Dzeletovic et. al. (modified) [9]. Internal standard (100 ng of 24-hydroxycholesterol-d7 and 27- hydroxycholesterol-d7 in chloroform; Avanti Polar Lipids, Alabaster, USA) was added in samples and oxysterols measurements were performed after alkaline hydrolysis adding a mixture of $10 \mathrm{~mL}$ of absolute ethanol and $0.4 \mathrm{M}$ of potassium hydroxide overnight, at room temperature. The $\mathrm{pH}$ was adjusted to 7 with phosphoric acid followed by $20 \mathrm{~mL}$ of chloroform and $6 \mathrm{~mL}$ of water. After vigorous shaking and centrifugation at $4 \circ \mathrm{C}$, the aqueous phase was removed and the organic phase evaporated. The lipid extract was dissolved in toluene $(1 \mathrm{~mL})$. Oxysterols were separated from cholesterol by solid phase extraction. Briefly, the sample was applied into the column (Sigma-Aldrich Supelclean LC-Si SPE Tubes SUPELCO, Bellefonte, USA) previously conditioned with $8 \mathrm{~mL}$ of hexane. Cholesterol were eluted with 1.5\% isopropanol in hexane $(8 \mathrm{~mL})$, and oxysterols were further eluted with $30 \%$ isopropanol in hexane $(6 \mathrm{~mL})$. Finally, the solvent was evaporated and samples were derivatized with $100 \mu \mathrm{L}$ of pyridine and $100 \mu \mathrm{L}$ of N,O-bis (trimethylsilyl) trifluoroacetamide with trimethylchlorosilane (BSTFA; Sigma- Aldrich, St. Louis, USA), for $1 \mathrm{~h}$ at 60 ॰C. The derivatized sample $(1 \mu \mathrm{L})$ was injected into a gas chromatograph coupled to a mass spectrometer (Shimadzu GCMS-QP2010, Kyoto, Japan) by automatic injector and analyzed in selected ion monitoring. The separation was performed on a Restek capillary column (100\% dimethyl polysiloxane-RxiR $-1 \mathrm{~ms}$. Cat. \#13323), $30 \mathrm{~m}$, internal diameter $0.25 \mathrm{~mm}$, for $30 \mathrm{~min}$, using helium as mobile phase, with constant linear velocity of $44.1 \mathrm{~cm} / \mathrm{sec}$. The oven started at $240 \circ \mathrm{C}$ with increment of $5 \circ \mathrm{C} / \mathrm{min}$, for $7 \mathrm{~min}$ up to 
290 . C. The mass spectrometer operated in impact electron mode at an ionization voltage of $70 \mathrm{eV}$ with the temperature of the ion source at $300 \circ \mathrm{C}$.

The quantification was performed comparing the peak areas of the standard curve and corrected for internal standards [10]. Plasma non-cholesterol sterols $(\mu \mathrm{g})$ and oxysterols $(\mathrm{ng})$ were expressed as ratio of plasma total cholesterol (mg).

\section{Statistical analysis}

The results were expressed as mean $\pm S D$ or the median (variation). Differences between groups were compared by Kruskal Wallis $(p<0.05)$ and Dunn's multiple comparison with correction by Bonferroni post hoc $(p<0.017)$ was performed when appropriate. Different letters represent statistically significant in the post-test. Gender distribution were compared by Chi-squared test $(p<0.05)$

\section{Results}

Anthropometric data, glucose, lipids and plasma lipoproteins concentrations, characterizing the samples are presented in Table 1. Characterizing each group HDL-C plasma concentrations showed significant differences. Triglycerides and VLDL-C plasma levels are lower in the High HDL group when compared to the Low HDL and the Control group. Triglycerides were within the normal range ( $<150 \mathrm{mg} / \mathrm{dL}$ ) in all participants. 


\begin{tabular}{|c|c|c|c|c|}
\hline & Low HDL & High HDL & Control & $\mathrm{p}$ \\
\hline $\mathrm{n}$ & 12 & 15 & 10 & \\
\hline Gender (men/women) & $7 / 5$ & $7 / 8$ & $4 / 6$ & \\
\hline Age (years) & $46 \pm 14$ & $52 \pm 14$ & $47 \pm 11$ & \\
\hline BMI $\left(k g / m^{2}\right)$ & $24 \pm 2$ & $22 \pm 2$ & $23 \pm 3$ & \\
\hline Glucose (mg/dL) & $89 \pm 11$ & $89 \pm 7$ & $89 \pm 5$ & \\
\hline Cholesterol (mg/dL) & $163 \pm 28$ & $198 \pm 37$ & $173 \pm 44$ & \\
\hline $\mathrm{HDL}-\mathrm{C}(\mathrm{mg} / \mathrm{dL})$ & $34(25-39)$ a & $74(62-95) b$ & $46(42-49) \mathrm{c}$ & $<0.001$ \\
\hline LDL-C (mg/dL) & $104 \pm 17$ & $108 \pm 31$ & $107 \pm 39$ & \\
\hline VLDL-C (mg/dL) & $21 \pm 6 a$ & $14 \pm 6 b$ & $22 \pm 9 a$ & 0.009 \\
\hline Triglycerides (mg/dL) & $107 \pm 30 a$ & $71 \pm 29 b$ & $108 \pm 44 a$ & 0.011 \\
\hline \multicolumn{5}{|c|}{$\begin{array}{l}\text { BMI (body mass index), VLDL-C (very-low-density lipoproteins cholesterol), LDL-C (low-density } \\
\text { lipoproteins cholesterol), HDL-C (high-density lipoproteins cholesterol). Kruskal Wallis }(P<0.05) \text {, } \\
\text { Dunn's post-test with correction by Bonferroni. Different letters represent statistically significant in the } \\
\text { post-test. }\end{array}$} \\
\hline
\end{tabular}

To eliminate the influence of cholesterol concentrations between individuals, as well as in their lipoproteins, plasma oxysterols were expressed in relation to cholesterol [11].

24-OHC plasma levels were higher in the Low HDL group $(p=0.024)$ compared to High HDL, but the percentage distribution in lipoproteins did not differ between groups (Table 2). 27-OHC plasma levels and the percentage distribution in lipoproteins did not differ between groups (Table 3$)(p=0.07)$. 
Table 2

24-OHC (ng) to cholesterol $(\mathrm{mg})$ ratio in plasma and its percentage distribution among lipoproteins. The results were expressed as the median (variation).

\begin{tabular}{|lllll|}
\hline & Low HDL & High HDL & Control & P \\
\hline $\mathrm{n}$ & 12 & 15 & 10 & 0.024 \\
\hline Plasma (ng/mg) & 75 & 52 & 63 & \\
& $(46-198) \mathrm{a}$ & $(34-100) \mathrm{b}$ & $(9-120)$ ab & \\
\hline Percentage distribution (\%) & & & $20(6-40)$ & \\
VLDL & $14(9-19)$ & $10(6-39)$ & $28(11-54)$ & \\
\hline LDL & $27(16-70)$ & $35(14-49)$ & $38(12-64)$ & \\
\hline HDL & $32(11-66)$ & $35(16-68)$ & $(6-31)$ & \\
\hline LFF & $11(0-45)$ & $13(3-37)$ & & \\
\hline $\begin{array}{l}\text { VLDL (very-low-density lipoproteins), LDL (low-density lipoproteins), HDL (high-density lipoproteins) } \\
\text { and LFF (lipid free fraction). Kruskal Wallis (P< } 0.05), \text { Dunn's post-test with correction by Bonferroni. } \\
\text { Different letters represent statistically significant in the post-test. }\end{array}$ & \\
\hline
\end{tabular}

Table 3

$27-\mathrm{OHC}(\mathrm{ng})$ to cholesterol $(\mathrm{mg})$ ratio in plasma and its percentage distribution among lipoproteins. The results were expressed as the median (variation).

\begin{tabular}{|lllll|}
\hline & Low HDL & High HDL & Control & p \\
\hline Plasma (ng/mg) & 113 & 86 & 123 & 0.07 \\
& $(54-141)$ & $(48-151)$ & $(69-323)$ & \\
\hline Percentage distribution (\%) & & & $19(0-35)$ \\
VLDL & $19(14-47)$ & $9(0-26)$ & $41(21-42)$ \\
\hline LDL & $36(23-54)$ & $36(19-64)$ & $40(26-60)$ \\
\hline HDL & $36(30-50)$ & $42(28-64)$ & $0(0-17)$ & \\
\hline LFF & $0(0-0)$ & $0(0-38)$ & \\
\hline $\begin{array}{l}\text { VLDL (very-low-density lipoproteins), LDL (low-density lipoproteins), HDL (high-density lipoproteins) } \\
\text { and LFF (lipoproten free fraction). Kruskal Wallis (P< } 0.05), \text { Dunn's post-test with correction by } \\
\text { Bonferroni. Different letters represent statistically significant in the post-test. }\end{array}$ \\
\hline
\end{tabular}

NCSPCS (desmosterol and lathosterol) and phytosterols (campesterol and sitosterol) plasma levels properly corrected cholesterol concentration (expressed as $\mu \mathrm{g} / \mathrm{mg}$ cholesterol) did not differ among groups (Tables 4, 5, 6 and 7). 
Percentage of NCSPCS and phytosterols are predominan in LDL, which carries about $50 \%$ of these molecules (Tables 4, 5, 6 and 7), but did not differ between groups. $30 \%$ of desmosterol and lathosterol are present in $\mathrm{HDL}$, with the High HDL group having significantly higher percentage than the Low HDL and Control group (Tables 4 and 5). VLDL transports less than 20\% of NCSPCS. Percent lathosterol was lower in the High HDL group than in the Low HDL and Control groups (Table 5). $30 \%$ of campestrol and sitosterol are present in the High HDL group being significantly higher than in the Low HDL and Control groups. Conversely, the sitosterol LDL distribution favored the Low HDL and Control groups (Tables 6 and 7).

\section{Table 4. Desmosterol $(\mathrm{ng})$ to cholesterol $(\mathrm{mg})$ ratio in plasma and its percentage distribution among} lipoproteins. The results were expressed as the median (variation).

\begin{tabular}{|lllll|}
\hline & Low HDL & High HDL & Control & $p$ \\
\hline Plasma $(\mu \mathrm{g} / \mathrm{mg})$ & 0.770 & 0.633 & 0.508 & \\
& $(0.250-1.715)$ & $(0.053-1.431)$ & $(0.209-1.321)$ & \\
\hline Percentage distribution $(\%)$ & & & \\
\hline VLDL & $17(9-39)$ & $12(0-38)$ & $15(3-23)$ & 0.039 \\
\hline LDL & $54(24-77)$ & $55(22-92)$ & $64(43-77)$ & 24 (14-40) \\
\hline HDL & $26(7-40)$ & $33(8-64)$ & & \\
\hline $\begin{array}{l}\text { VLDL (very-low-density lipoproteins), LDL (low-density lipoproteins), HDL (high-density lipoproteins). } \\
\text { Kruskal Wallis (P<0.05), Dunn's post-test with correction by Bonferroni. Different letters represent } \\
\text { statistically significant in the post-test. }\end{array}$ & & & \\
\hline
\end{tabular}

Table 5. Lathosterol (ng) to cholesterol ( $\mathrm{mg}$ ) ratio in plasma and its percentage distribution among lipoproteins. The results were expressed as the median (variation).

\begin{tabular}{|c|c|c|c|c|}
\hline & Low HDL & High HDL & Control & $\mathrm{p}$ \\
\hline $\mathrm{n}$ & 12 & 15 & 9 & \\
\hline \multirow[t]{2}{*}{ Plasma $(\mu \mathrm{g} / \mathrm{mg})$} & 0.188 & 0.159 & 0.272 & \\
\hline & $(0.043-0.467)$ & $(0.068-0.327)$ & $(0.082-0.534)$ & \\
\hline \multicolumn{5}{|c|}{ Percent distribution (\%) } \\
\hline VLDL & $19(11-55) a$ & $14(4-37) b$ & $18(11-31) a, b$ & 0.041 \\
\hline LDL & $56(29-76)$ & $56(17-69)$ & $62(49-68)$ & \\
\hline HDL & $16(5-56) a$ & $29(20-59) b$ & $20(11-38) a, b$ & 0.006 \\
\hline \multicolumn{5}{|c|}{$\begin{array}{l}\text { VLDL (very-low-density lipoproteins), LDL (low-density lipoproteins), HDL (high-density lipoproteins). } \\
\text { Kruskal Wallis }(P<0.05) \text {, Kruskal Wallis }(P<0.05) \text {, Dunn's post-test with correction by Bonferroni. } \\
\text { Different letters represent statistically significant in the post-test. }\end{array}$} \\
\hline
\end{tabular}


Table 6. Campesterol $(\mathrm{ng})$ to cholesterol $(\mathrm{mg})$ ratio in plasma and its percentage distribution among lipoproteins. The results were expressed as the median (variation).

\begin{tabular}{|lllll|}
\hline & Low HDL & High HDL & Control & $p$ \\
\hline $\mathrm{n}$ & 12 & 15 & 10 & 0.933 \\
\hline Plasma $(\mu \mathrm{g} / \mathrm{mg})$ & 1.437 & 1.066 & $(0.220-2.077)$ \\
& $(0.375-2.713)$ & $(0.406-3.019)$ & & 0.003 \\
\hline Percent distribution (\%) & $20(12-50)$ & $18(3-33)$ & $17(8-35)$ \\
\hline VLDL & $58(37-70)$ & $54(22-61)$ & $56(47-63)$ \\
\hline LDL & $31(9-43)$ a & $39(26-51)$ b & 38 (16-44) a, b \\
\hline HDL & & & \\
\hline $\begin{array}{l}\text { VLDL (very-low-density lipoproteins), LDL (low-density lipoproteins), HDL (high-density lipoproteins. } \\
\text { Kruskal Wallis (P<0.05), Dunn's post-test with correction by Bonferroni. Different letters represent } \\
\text { statistically significant in the post-test. }\end{array}$ & & \\
\hline
\end{tabular}

\section{Table 7. Sitosterol (ng) to cholesterol ( $\mathrm{mg}$ ) ratio in plasma and its percentage distribution among} lipoproteins. The results were expressed as the median (variation).

\begin{tabular}{|lllll|}
\hline & Low HDL & High HDL & Control & $\mathrm{p}$ \\
\hline $\mathrm{n}$ & 12 & 15 & 9 & \\
\hline Plasma $(\mu \mathrm{g} / \mathrm{mg})$ & 1.244 & 1.402 & 0.949 & \\
& $(0.496-2.988)$ & $(0.396-2.447)$ & $(0.204-2.437)$ & \\
\hline Percent distribution (\%) & $15(10-51)$ & $10(2-65)$ & $15(9-35)$ & 0.028 \\
\hline VLDL & $60(34-71)$ a & $47(21-55)$ b & $51(36-65)$ a, b & 0.002 \\
\hline LDL & $23(9-50)$ a & $43(11-67)$ b & 33 (19-42) a, b & \\
\hline HDL & & & & \\
\hline $\begin{array}{l}\text { VLDL (very-low-density lipoproteins), LDL (low-density lipoproteins), HDL (high-density lipoproteins). } \\
\text { Kruskal Wallis (P< } 0.05), \text { Dunn's post-test with correction by Bonferroni. Different letters represent } \\
\text { statistically significant in the post-test. }\end{array}$ & & & \\
\hline
\end{tabular}

\section{Discussion}

The percentage distribution of oxysterols among lipoproteins in High HDL, Low HDL and Controls was reported in healthy volunteers with similar plasma $\mathrm{HDL}-\mathrm{C}[5,12]$. Our results agree with the study showing 24- $\mathrm{OHC} /$ cholesterol and $27-\mathrm{OHC} /$ cholesterol ratios higher in the HDL fraction, indicating the importance of HDL for the transport of these oxysterols [5]. However, we found lower 24-OHC (0.024), (borderline 27- 
$\mathrm{OHC}, \mathrm{p}=0.07$ ) plasma level in subjects with High HDL, which suggests that the increase in HDL does not provide greater efficiency for cellular export of cholesterol metabolites.

On the other hand, we found no percent differences between groups regarding the distribution of NCSPCS among lipoproteins. This is probably due to the fact that this distribution is likely dependent on other factors such as their esterification rate by the enzyme lecithin-cholesterol acyltransferase (LCAT) and transfer between lipoproteins mediated by cholesteryl ester transfer protein (CETP) [13]. In this regard LCAT activity is higher in individuals with low HDL-C plasma concentration and the CETP activity does not differ between individuals with low and high plasma HDL-C levels [14]. VLDL transports less than $20 \%$ of NCSPCS. Approximately $30 \%$ of desmosterol and lathosterol are present in HDL. The High HDL group carried higher NCSPCS percent than the other groups. Although the fraction of these sterols transported in LDL is the largest, we noted that participation of HDL increase suggesting its role in the cellular removal of cholesterol synthesis precursors.

Similarly to cholesterol, phytosterols and phytostanols are carried in lipoproteins, being $70-80 \%$ in low density lipoprotein (LDL) and $20-30 \%$ in high density lipoprotein (HDL) $[15,16]$. Simonem et al. (2007) showed in normal weight, good to moderate glucose balance, no insulin therapy, mild to moderate hypercholesterolemia, and normotriglyceridemia type 2 diabetics campesterol and sitosterol concentrations $7 \%-9 \%$ in VLDL, 3\%-4\% in IDL, $59 \%-61 \%$ in LDL, and $27 \%-30 \%$ in HDL [17]. We found similar distributions of phytosterols as shown by Björkhem et al. [15, 16] and Simonem et al.[17], however our High HDL-C group presented higher percent campesterol and sitosterol in HDL, suggesting that phytosterols absorbed in the enterocytes are incorporated into the nascent HDL. Similar observations of higher plasma phytosterol levels have been made in patients with high HDL-C levels (matched for similar LDL-C levels) [4] and patients with high HDL-C levels due to exercise [18], although in the PROCAM study (Prospective Cardiovascular Münster), patients with low HDL-C levels displayed decreased plasma phytosterol and a direct correlation occurred between low HDL-C and decreased plasma phytosterol [19]. However, PROCAM dealt with metabolic syndrome, not with normal cases.

It is worth noting that the increase in phytosterols in cases of elevated HDL-C caused by the CETP inhibitor dalcetrapib is similar to that seen with statin treatment [20] and different from that measured in ABCG5/G8 mutation leading to atherogenic phytosterolemia [21]. Based on these results it was proposed that phytosterols not returned to the intestinal lumen via ABCG5/G8 activity are absorbed via chylomicrons with trace amounts absorbed via an HDL pathway and very likely efficiently excreted by the liver [22]. However, since measurable amounts of cholesterol are absorbed at the intestinal level through the ABCA1/ApoA1 efflux system [23] it has been hypothesized that the absorption of phytosterol - which cannot be synthetized by animals - via the HDL pathway could be used as a marker of intestinal ABCA1/ApoA1 activity [24]. Accordingly, Niesor E.J et al. showed that plant sterols, which offer the advantage of being strictly of dietary origin, are absorbed at the intestinal level via an HDL pathway [25], very likely due to ApoA1 lipidation with cholesterol, and related to pre-beta-1 HDL levels [26]. The original observation [25] was made both in hamsters and healthy human volunteers treated with the CETP modulator dalcetrapib, which affects HDL metabolism in both species. High percent of HDL campesterol 
and sitosterol suggests phytosterols absorbed in the enterocytes mostly are incorporated into the nascent $\mathrm{HDL}$ and corroborates the hypothesis of Niesor EJ et al. [24] that phytosterol absorption via the HDL pathway could represent a marker of intestinal ABCA1/ApoA1 activity. An indirect proof of this concept is the lack of HDL-increase in plasma phytosterol on CETP inhibition with dalcetrapib in patients with mutations in ApoA1 and/or ABCA1 [27].

\section{Conclusion}

Elevated percentage HDL desmosterol and lathosterol in the High HDL group suggests HDL facilitates the export of these NCSPCS from cells but not the export of the cholesterol metabolite 24-OHC which was lower in the High HDL than in the Low HDL group. A high percentage of campesterol and sitosterol in $\mathrm{HDL}$ suggests that phytosterols are absorbed by enterocytes, incorporated mainly into nascent HDL and corroborates the hypothesis of Niesor EJ et al. [24] that the absorption of phytosterol via the HDL pathway could be used as a marker of the intestinal activity of ABCA1 / ApoA1.

\section{Abbreviations}

\section{4-OHC}

24-hydroxycholesterol

27-OHC

27-hydroxycholesterol

\section{ABCA1}

ATP Binding Cassette Subfamily A Member 1

ABCG5/G8

ATP-Cassette Binding Proteins G5/G8

ApoA1

Apolipoproteína A-I

BHT

butylated hydroxytoluene

BMI

body mass index

BSTFA

N,O-bis (trimethylsilyl) trifluoroacetamide

CETP

Cholesteryl Ester Transfer Protein

\section{EDTA}

ethylenediamine tetraacetic acid

HDL-C

High-Density Lipoproteins-Cholesterol

\section{LCAT}


Lecithin-Cholesterol Acyltransferase

LDL

low-density lipoproteins (), high-density lipoproteins (HDL)

LFF

lipid free fraction

NCSPCS

Noncholesterol Sterols Precursors of Cholesterol Synthesis

PROCAM

Prospective Cardiovascular Münster

TMCS

trimethylchlorosilane

VLDL

very-low-density lipoproteins

\section{Declarations}

\section{Ethics approval and consent to participate}

This study was approved by the Research Ethics Committee of UNICAMP School of Medicine under $n^{\circ}$ 120/2007 and Hospital das Clínicas da Faculdade de Medicina da Universidade de São Paulo under nº 149/7. All participants were informed of the objectives of the protocol and signed an informed written consent according to research protocols approved by the Ethics Committee of HCFMUSP and UNICAMP.

\section{Consent for publication}

All the authors have consented for the publication of this study.

\section{Availability of data and materials}

The data used to support the findings of this study are included within the article. Additional data or information can be requested by contacting the corresponding author.

\section{Competing interests}

The authors declare that they have no competing interests"

\section{Funding}

Grant support: FAPESP (São Paulo Research Foundation) research project 06/60585-9 and fellowship support to Eliton Junior da Silva (2013/13631-9)

\section{Authors' contributions}


VSN: Conceptualization, Methodology, Funding acquisition, Writing - Review \& Editing. EJS: Investigation, Writing - Original Draft. GSF: Formal analysis. SISA: Investigation. VHSZ: Data Curation. PMC: Investigation, Writing - Original Draft. ERN: Writing - Review \& Editing. ECF: Writing - Review \& Editing , Data Curation. ECRQ: Supervision, Writing - Review \& Editing

\section{Acknowledgements}

Not applicable

\section{References}

1. Miettinen TA, Tilvis RS, Kesaniemi YA. Serum plant sterols and cholesterol precursors reflect cholesterol absorption and synthesis in volunteers of a randomly selected male population. American journal of epidemiology United States. 1990;131:20-31.

2. Tilvis RS, Miettinen TA. Serum plant sterols and their relation to cholesterol absorption. The American journal of clinical nutrition United States. 1986;43:92-7.

3. Miettinen TA, Tilvis RS, Kesaniemi YA. Serum cholestanol and plant sterol levels in relation to cholesterol metabolism in middle-aged men. Metabolism: clinical experimental United States. 1989;38:136-40.

4. Nunes VS, Leança CC, Panzoldo NB, Parra E, Cazita PM, Nakandakare ER, et al. HDL-C concentration is related to markers of absorption and of cholesterol synthesis: Study in subjects with low vs. high HDL-C. Clinica Chimica Acta. 2011;412.

5. Babiker A, Diczfalusy U. Transport of side-chain oxidized oxysterols in the human circulation. Biochimica et biophysica acta. Netherlands. 1998;1392:333-9.

6. Nunes VS, Leança CC, Panzoldo NB, Parra E, Zago V, Cazita PM, et al. Plasma 27-

hydroxycholesterol/cholesterol ratio is increased in low high density lipoprotein-cholesterol healthy subjects. Clinical Biochemistry. 2013;46.

7. Parra ES, De Souza Zago VH, Panzoldo NB, Alexandre F, Vendrame F, De Moura Virginio VW, et al. Development of a clinical laboratory data base of hyper and hypo alpha lipoproteins in Campinas-SP and neighboring region. Jornal Brasileiro de Patologia e Medicina Laboratorial. 2013;49.

8. HAVEL RJ, EDER HA, BRAGDON JH. The distribution and chemical composition of ultracentrifugally separated lipoproteins in human serum. The Journal of clinical investigation United States. 1955;34:1345-53.

9. Dzeletovic S, Breuer O, Lund E, Diczfalusy U. Determination of cholesterol oxidation products in human plasma by isotope dilution-mass spectrometry. Analytical biochemistry United States. 1995;225:73-80.

10. Ferreira GS, Pinto PR, Iborra RT, Del Bianco V, Santana MFM, Nakandakare ER, et al. Aerobic exercise training selectively changes oxysterol levels and metabolism reducing cholesterol accumulation in the aorta of dyslipidemic mice. Frontiers in Physiology. 2017;8. 
11. Miettinen TA. Detection of changes in human cholesterol metabolism. Annals of clinical research Finland. 1970;2:300-20.

12. Burkard I, von Eckardstein A, Waeber G, Vollenweider P, Rentsch KM. Lipoprotein distribution and biological variation of 24S- and 27-hydroxycholesterol in healthy volunteers. Atherosclerosis Ireland. 2007;194:71-8.

13. Lin CY, Morel DW. Esterification of oxysterols in human serum: effects on distribution and cellular uptake. Journal of lipid research United States. 1996;37:168-78.

14. Leança CC, Nunes VS, Panzoldo NB, Zago VS, Parra ES, Cazita PM, et al. Metabolism of plasma cholesterol and lipoprotein parameters are related to a higher degree of insulin sensitivity in high HDL-C healthy normal weight subjects. Cardiovascular Diabetology. 2013.

15. Ingemar B; Kirsten Muri Boberg; Eran Leitersdorf. Inborn errors in bile acid biosynthesis and storage of sterols other than cholesterol. The Metabolic and Molecular Bases of Inherited Disease [Internet]. 2001. p. 2961-2988. Available from: https://ommbid.mhmedical.com/.

16. Gylling H, Simonen P. Phytosterols. Phytostanols, and Lipoprotein Metabolism. Nutrients Switzerland. 2015;7:7965-77.

17. Simonen PP, Gylling H, Miettinen TA. The distribution of squalene and non-cholesterol sterols in lipoproteins in type 2 diabetes. Atherosclerosis Ireland. 2007;194:222-9.

18. Wilund KR, Feeney LA, Tomayko EJ, Weiss EP, Hagberg JM. Effects of endurance exercise training on markers of cholesterol absorption and synthesis. Physiological research. Czech Republic. 2009;58:545-52.

19. Assmann G, Cullen P, Kannenberg F, Schulte H. Relationship between phytosterol levels and components of the metabolic syndrome in the PROCAM study. European journal of cardiovascular prevention and rehabilitation: official journal of the European Society of Cardiology, Working Groups on Epidemiology \& Prevention and Cardiac Rehabilitation and Exercise Physiology. England. 2007;14:208-14.

20. Matthan NR, Giovanni A, Schaefer EJ, Brown BG, Lichtenstein AH. Impact of simvastatin, niacin, and/or antioxidants on cholesterol metabolism in CAD patients with low HDL. Journal of lipid research United States. 2003;44:800-6.

21. Patel SB. Plant sterols and stanols: their role in health and disease. Journal of clinical lipidology United States. 2008;2:11-9.

22. Robins SJ, Fasulo JM. High density lipoproteins, but not other lipoproteins, provide a vehicle for sterol transport to bile. The Journal of clinical investigation United States. 1997;99:380-4.

23. Hussain MM. Intestinal lipid absorption and lipoprotein formation. Current opinion in lipidology. England. 2014;25:200-6.

24. Niesor EJ. Will Lipidation of ApoA1 through Interaction with ABCA1 at the Intestinal Level Affect the Protective Functions of HDL? Biology. Switzerland. 2015;4:17-38.

25. Niesor EJ, Chaput E, Staempfli A, Blum D, Derks M, Kallend D. Effect of dalcetrapib, a CETP modulator, on non-cholesterol sterol markers of cholesterol homeostasis in healthy subjects. 
Atherosclerosis Ireland. 2011;219:761-7.

26. Niesor EJ, Gauthamadasa K, Silva RAGD, Suchankova G, Kallend D, Gylling H, et al. Xanthophylls, phytosterols and pre-beta1-HDL are differentially affected by fenofibrate and niacin HDL-raising in a cross-over study. Lipids United States. 2013;48:1185-96.

27. Niesor EJ, Kallend D, Bentley D, Kastelein JJP, Kees Hovingh G, Stroes ESG. Treatment of low HDL-C subjects with the CETP modulator dalcetrapib increases plasma campesterol only in those without ABCA1 and/or ApoA1 mutations. Lipids United States. 2014;49:1245-9. 